\title{
Diabetes and premature menopause: is their co-existence detrimental to the skeleton?
}

\author{
D J Hadjidakis, A M Mylonakis, M E Sfakianakis, A E Raptis and S A Raptis \\ 2nd Department of Internal Medicine-Propaedeutic, Research Institute and Diabetes Centre, Athens University, 'Attikon' and 'Evgenidion' Hospitals, \\ Athens, Greece
}

(Correspondence should be addressed to D J Hadjidakis, 2nd Department of Internal Medicine-Propaedeutic and Research Institute, Athens University, PO Box 171 51, Athens 100 24, Greece; Email: dhadjida@med.uoa.gr)

\begin{abstract}
Objective: Premature menopause is a known risk factor for osteoporosis, whilst the influence of type 2 diabetes on bone mineral density (BMD) is still controversial.

Design and methods: BMD values assessed by dual-energy X-ray absorptiometry (DXA) in L2-L4 vertebrae and the femoral neck (FN) of 40 diabetic women with premature menopause (D-EMP) were compared with those of 60 non-diabetic, prematurely menopausal women (EMP) and 60 diabetic women with normal menopause (D-NMP) who had been matched by age and body mass index (BMI). In all women, the time elapsed since menopause ranged between 10 and 25 years and the duration of diabetes exceeded $75 \%$ of the postmenopausal time period. The age of D-EMP women was $58.7 \pm 5$ years (mean \pm 1 S.D.), age at menopause $39.5 \pm 2.7$, years since menopause $18.6 \pm 4.9$, BMI $27.8 \pm 4.3 \mathrm{~kg} / \mathrm{m}^{2}$ and duration of diabetes $13.9 \pm 3.9$ years.

Results: Vertebral BMD values of D-EMP women were significantly higher than those of EMP women $\left(0.908 \pm 0.135\right.$ vs. $\left.0.817 \pm 0.14 \mathrm{~g} / \mathrm{cm}^{2}, P=0.002\right)$, although there was no significant difference between D-EMP and D-NMP women $\left(0.886 \pm 0.15 \mathrm{~g} / \mathrm{cm}^{2}\right)$. No significant differences were observed in FN BMD values between all groups. Age-adjusted BMD values ( $\mathrm{Z}$ scores) of D-EMP women were higher than EMP women in both anatomic sites $(P<0.01)$, but did not differ from D-NMP women. In contrast to the other two groups, no statistically significant correlation was observed in D-EMP women between the BMD values of either anatomic area and the time elapsed since menopause. $\mathrm{HbA}_{1 \mathrm{c}}$ values were positively correlated only to vertebral BMD values of the D-EMP group $(P<0.05)$. No correlation was observed between the BMD values and the duration of diabetes either in D-EMP or in D-NMP women.

Conclusions: Type 2 diabetes seems to positively affect the mineral density of the trabecular bone in women with premature menopause. The duration of diabetes does not appear to influence bone mass.
\end{abstract}

European Journal of Endocrinology 152 437-442

\section{Introduction}

Osteoporosis is a silent epidemic that constitutes a great socio-economic problem, with a negative impact both on morbidity and mortality. Hip fracture is associated with a $12 \%$ increase in mortality rate over the subsequent first year. Studies on bone mineral density (BMD) in diabetic patients have given inconsistent results. More specifically, type 2 diabetes has been reported to be associated with decreased (1), unchanged $(2,3)$ or increased (4) BMD values. The issue is further complicated when it is taken into consideration that the incidence of fractures representing the clinical outcome of osteoporosis were found to be either increased $(5,6)$, unchanged $(7)$ or decreased (4) in type 2 diabetics compared with non-diabetic persons.
Bone loss in either osteopenia or osteoporosis is multifaceted, with age and ovarian function being the crucial factors, although race, heredity and life-style also contribute $(8-11)$. Bone mass status after menopause generally depends on the composition and the degree of mechanical loading. The loss of bone mass is accelerated after the cessation of ovarian secretion and its magnitude depends on the type and time of menopause $(11-14)$. Premature menopause, either spontaneous or surgical due to bilateral oophorectomy, represents an established risk factor for osteoporosis and is associated with an increased frequency of hip fractures (15).

Type 1 and type 2 diabetes, as two distinct entities of different pathogenesis, exert a non-uniform influence on bone metabolism. In both cases, a lack of correlation of biochemical and densitometry parameters to the degree of glucose control has often been demonstrated. 
In the past, various studies have illustrated a positive effect of type 2 diabetes on bone mass (16-18) and in an attempt to explain this finding, investigators proposed specific pathophysiological mechanisms such as obesity, hyperinsulinaemia (16), or increased concentrations of growth factors (insulin-like growth factors (IGFs)) (17). Up to now, studies that have addressed the influence of type 2 diabetes on the bone metabolism of postmenopausal women have mainly dealt with normal menopause. Premature menopause has been reported as being associated with type 1 diabetes (19), although such an association has not been verified regarding type 2 diabetes (20). The investigation of the combination of type 2 diabetes exerting a controversial, independent effect and premature menopause having a known, negative effect on bone metabolism might yield important findings.

In the present study, we investigated the BMD status of diabetic women with premature menopause, well in advance of their menopausal age. This was compared with the same status of diabetic women after normal menopause and with non-diabetic women with spontaneous menopause. Our comparison dealt with two anatomic areas of different bone composition, the vertebrae (mainly trabecular) and the femoral neck (mixed trabecular-cortical).

\section{Subjects and methods}

\section{Subjects}

Diabetic postmenopausal women and non-diabetic, prematurely postmenopausal women are routinely screened for osteoporosis in our Outpatient Clinic for Metabolic Bone Diseases. The diabetic population were derived from the Diabetes Outpatient Clinic belonging to our department. From an initial population of 550 postmenopausal women with type 2 diabetes, 40 premature postmenopausal women were identified and studied (D-EMP). Two other groups, featuring 60 non-diabetic women with premature menopause (EMP) and 60 type 2 diabetic women after normal menopause (D-NMP), were matched by age, body mass index (BMI), and glycosylated haemoglobin $\left(\mathrm{HbA}_{1 \mathrm{c}}\right)$ levels with the D-EMP women, and served as control groups. Exclusion criteria for all women were the presence of any disease or use of medication (including hormone replacement therapy) that has a known influence on bone metabolism, an inadequate calcium intake (less than $600 \mathrm{mg}$ daily) and restricted daily activity. All information was obtained using a standard questionnaire routinely completed by all subjects examined in our clinic. The presence of late diabetic complications, apart from mild retinopathy, also precluded participation in this study. The cut-off age used for the definition of premature menopause was 42 years, selected on the basis of 2 standard deviations below the average age of menopause of the Hellenic
Table 1 Anthropometric characteristics of the three groups of women studied (results are means \pm 1 s.D.). D-NMP women were of similar chronological age, but with fewer years elapsed since menopause.

\begin{tabular}{lccc}
\hline & $\begin{array}{c}\text { EMP } \\
(n=60)\end{array}$ & $\begin{array}{c}\text { D-EMP } \\
(n=40)\end{array}$ & $\begin{array}{c}\text { D-NMP } \\
(n=60)\end{array}$ \\
\hline Age & $57.8 \pm 3.7$ & $58.7 \pm 5$ & $59.9 \pm 2.8$ \\
Menopausal age & $39.6 \pm 2.2$ & $39.9 \pm 2.7$ & $47.6 \pm 2.4$ \\
YSM & $18.2 \pm 3.8$ & $18.0 \pm 4.9$ & $13.0 \pm 1.9$ \\
BMI $\left(\mathrm{kg} / \mathrm{m}^{2}\right)$ & $26.9 \pm 2.8$ & $27.8 \pm 4.3$ & $27.7 \pm 5.1$ \\
$\mathrm{DUR}$ & & $13.9 \pm 3.9$ & $11.6 \pm 2.6$ \\
$\mathrm{HbA}{ }_{1} \mathrm{c}(\%)$ & & $6.5 \pm 0.8$ & $6.3 \pm 1$ \\
\hline
\end{tabular}

EMP, premature menopause; D-EMP, premature menopause with type 2 diabetes; D-NMP, normal menopause with type 2 diabetes; YSM, years since menopause; DUR, diabetes duration.

female population (21). For the categorisation of women in the premature menopausal groups (either diabetic or non-diabetic), we relied on self-reported ages of menopause. The period of time that had elapsed since menopause in all the women selected ranged between 10 and 25 years. The duration of diabetes in all diabetic women exceeded $3 / 4$ of the postmenopausal time period. Among the 40 D-EMP women, in 5 of them diabetes was diagnosed before or during the perimenopausal phase. Upon categorisation, we attempted to combine women in which a similar interval of time had elapsed since menopause into the 2 premature menopausal groups, while all women had a similar chronological age. The anthropometrical characteristics of the women studied are shown in Table 1. None of the women studied had ever received any type of hormone replacement therapy or any other medication with a known action on bone metabolism. Two D-EMP and 4 D-NMP women never required any medical treatment for the disease except from diet. Among the other 38 D-EMP women, five were receiving metformin, four sulphonylureas, eight metformin plus sulphonylureas, 16 insulin plus metformin and five insulin alone at the time of the study. In the remaining 56 women of the D-NMP group, 10 women were receiving metformin, seven sulphonylureas alone, 13 metformin plus sulphonylureas, 17 insulin plus metformin and nine insulin alone.

\section{Bone density measurements}

BMD was measured at the L2-L4 vertebrae (anteroposterior projection) and femoral neck with dual energy X-ray absorptiometry (DXA), using the Norland XR-26 Mark II densitometer (Norland Scientific Instruments, Fort Atkinson, WI, USA). The in vitro precision by repeated daily spine phantom measurements was $0.7 \%$, while the in vivo precision was $1.4 \%$, established in our laboratory by double measurements of 10 people at weekly intervals (21). 
BMD is expressed in $\mathrm{g} / \mathrm{cm}^{2}$ and in age-adjusted values (Z scores). The latter parameter represents the measure of standard deviations from the average age- and sex-matched normal population values. Estimation of the frequency of osteopenia or osteoporosis was based on the $\mathrm{T}$ score value, which represents the standard deviations of the individual BMD value from the BMD value of young sex-matched normal people from the Hellenic population (21). Osteopenia was defined as $-2.5<\mathrm{T}$ score $<-1$, and osteoporosis as $\mathrm{T}$ score $<-2.5$ (WHO criteria) (22). $\mathrm{HbA}_{1 \mathrm{c}}$ was measured using an automated HPLC analyser, HA-8140 (Menarini Diagnostics, Kyoto, Japan). Normal values ranged between 3.8 and $5.5 \%$.

\section{X-ray examination}

In all subjects, a lateral X-ray examination was carried out on the lumbar spine in order to exclude cases of diffuse inter-vertebral skeletal hyperostosis or degenerative lesions. The same investigator checked all X-rays.

\section{Statistics}

Normality of the data was tested according to the Kolmogorov-Smirnov test. Comparisons of absolute BMD values between the D-EMP, D-NMP and the EMP women were carried out according to Student's twotailed $t$-test and ANOVA. Pearson or Spearman correlation coefficients were calculated as appropriate to assess the type of association between either the duration of diabetes and $\mathrm{HbA}_{1 \mathrm{c}}$ levels or anthropometrical variables to BMD values. The stepwise multiple regression analysis was used in order to search for the influence of anthropometrical parameters on BMD behaviour. All anthropometrical characteristics, duration of diabetes and $\mathrm{HbA}_{1 \mathrm{c}}$ levels were tested as explanatory variables, since vertebral and femoral neck BMD values were stated as dependent variables. To compare the frequency of osteopenia and osteoporosis, the chi square equidistribution test was used. A $P$ value of less than 0.05 was considered statistically significant.

\section{Results}

D-EMP women exhibited significantly higher vertebral BMD values compared with the EMP women $\left(0.908 \pm 0.135\right.$ vs $\left.0.817 \pm 0.14 \mathrm{~g} / \mathrm{cm}^{2}, \quad P=0.002\right)$ whereas there was no significant difference in comparison with the D-NMP women $(0.908 \pm 0.135$ vs $0.886 \pm 0.15 \mathrm{~g} / \mathrm{cm}^{2}$ ) (Fig. 1). In contrast, FN BMD values did not differ significantly amongst the three groups (Fig. 1). Similar findings were obtained when comparing the BMI-adjusted BMD values of both anatomic sites between the three groups of women (Table 2). Age-adjusted BMD values (Z scores) did not differ significantly between the D-EMP and D-NMP

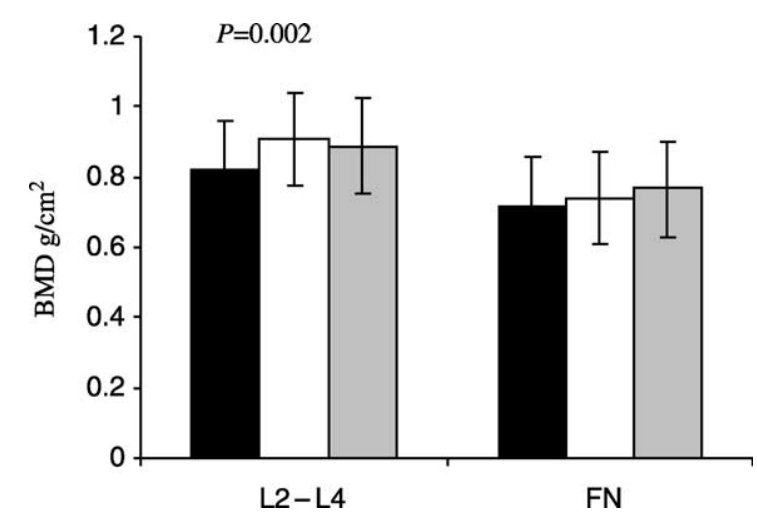

Figure $1 \mathrm{BMD}$ values of $\mathrm{L} 2-\mathrm{L} 4$ vertebrae and femoral neck (FN) in the three groups of women (means \pm 1 S.D.). Solid bars, EMP (premature menopause) women; open bars, D-EMP (premature menopause with type 2 diabetes) women; shaded bars, D-NMP (normal menopause with type 2 diabetes) women. L2-L4 BMD values of EMP women are significantly lower than the respective values of D-EMP women $(P=0.002$, Student's $t$-test).

Table 2 BMI-adjusted BMD values of L2-L4 vertebrae and the femoral neck (FN) between the three groups of women studied ( $\mathrm{g} / \mathrm{cm}^{2} /$ BMI unit $\times 10^{4}$, means \pm 1 S.D. $)$.

\begin{tabular}{lccccc}
\hline & $\begin{array}{c}\text { EMP } \\
(n=60)\end{array}$ & $\boldsymbol{P}$ & $\begin{array}{c}\text { D-EMP } \\
(n=40)\end{array}$ & $\boldsymbol{P}$ & $\begin{array}{c}\text { D-NMP } \\
(n=60)\end{array}$ \\
\hline L2-L4 & $306.2 \pm 57.6$ & 0.03 & $335.2 \pm 72.4$ & 0.47 & $325.2 \pm 58.8$ \\
FN & $254.7 \pm 44.5$ & 0.48 & $265.4 \pm 63.4$ & 0.37 & $278.3 \pm 54.9$
\end{tabular}

Vertebral BMD values of the EMP were significantly lower than those of the D-EMP groups.

groups, either in vertebrae or in $\mathrm{FN}$, whereas they were significantly greater in D-EMP compared with EMP women $(P=0.004$ for vertebrae and $P=0.01$ for FN) (Table 3). Based on the vertebral T score values, the proportions of women with osteopenia-osteoporosis were significantly lower in D-EMP compared with EMP women $(P<0.001)$. Between D-EMP and D-NMP women a trend in favour of the D-EMP group was observed regarding the same proportions (Fig. 2). The proportions based on FN T scores did not differ significantly between all the groups, although a trend for increased osteopenic-osteoporotic proportions was observed in the EMP group $(P=0.09)$ (Fig. 3).

In the D-EMP group, there was no significant correlation between the BMD values of either anatomic area

Table 3 Age-adjusted BMD values ( $Z$ scores) of the L2-L4 vertebrae and of femoral neck (FN) in the three groups of women studied (means \pm 1 S.D.).

\begin{tabular}{llllll}
\hline & \multicolumn{1}{c}{$\begin{array}{c}\text { EMP } \\
(n=60)\end{array}$} & $\boldsymbol{P}$ & $\begin{array}{c}\text { D-EMP } \\
(n=40)\end{array}$ & $\boldsymbol{P}$ & $\begin{array}{c}\text { D-NMP } \\
(\mathrm{n}=60)\end{array}$ \\
\hline L2-L4 & $-0.69 \pm 1.2$ & 0.004 & $0.14 \pm 1.3$ & 0.69 & $0.06 \pm 1.2$ \\
FN & $-0.48 \pm 0.89$ & 0.01 & $0.10 \pm 1.2$ & 0.77 & $0.14 \pm 1.1$
\end{tabular}

There were significantly lower $Z$ score values in the EMP than in the DEMP group, in both anatomic sites. 


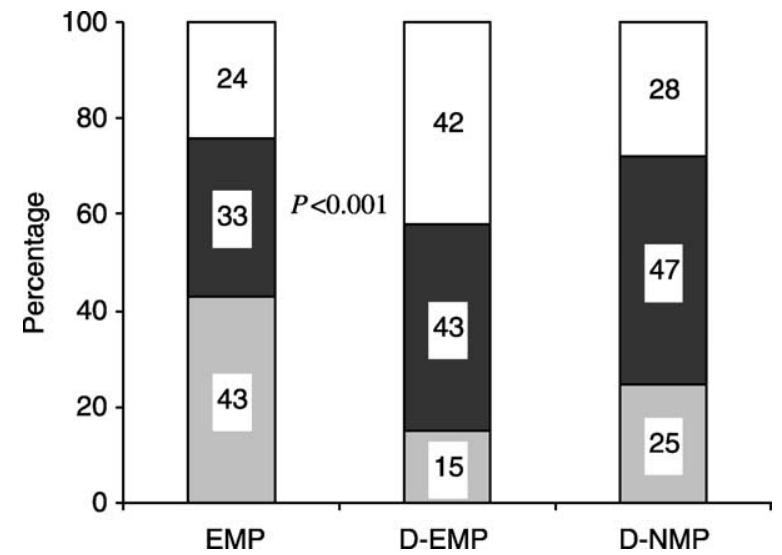

Figure 2 Percentages of osteoporosis (OPO; shaded bars) and osteopenia (OPE; solid bars) in the three groups of women based on vertebral (L2-L4) BMD T score values. Open bars, Normal $T$ score values above -1 . There is a significantly higher proportion of osteoporotic women in the EMP group compared with the D-EMP group $\left(P<0.001, \chi^{2}\right.$-test $)$.

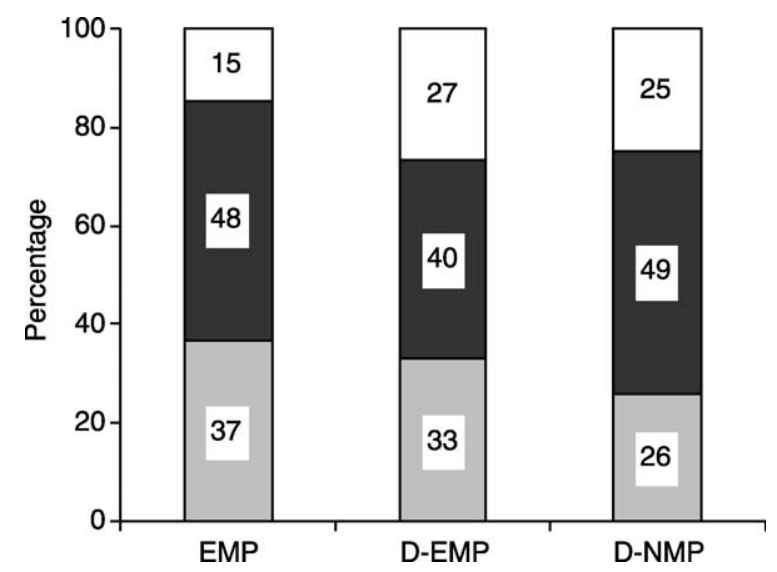

Figure 3 Percentages of osteoporosis (OPO; shaded bars) and osteopenia (OPE; solid bars) in the three groups of women based on femoral neck (FN) BMD T score values. Open bars, Normal $T$ score values above -1 . There are no significant differences between the groups $\left(\chi^{2}\right.$-test).

and the time period elapsed since menopause (Table 4). In contrast, a statistically significant inverse correlation was observed between FN BMD and the time since menopause in the EMP and D-NMP women, as well as between vertebral BMD and the time elapsed since menopause in the D-NMP women (Table 4). BMI and vertebral BMD were positively correlated in both diabetic groups $(P<0.05)$, whereas vertebral BMD was only positively correlated to $\mathrm{HbA}_{1 \mathrm{c}}$ in the D-EMP group $(P<0.05)$.

Applying stepwise multiple regression analysis, in the D-EMP women, glycosylated haemoglobin and chronological age in common explain $68 \%$ of the vertebral and $67 \%$ of the femoral neck BMD variance. In EMP women, chronological age combined with the number of years since menopause was found to explain $60 \%$ of the vertebral BMD variance, whereas age at menopause
Table 4 Correlation coefficients between BMD values and anthropometric or diabetic parameters in the three groups of women studied.

\begin{tabular}{lccc}
\hline Correlation & $\begin{array}{c}\text { EMP } \\
(n=60)\end{array}$ & $\begin{array}{c}\text { D-EMP } \\
(n=40)\end{array}$ & $\begin{array}{c}\text { D-NMP } \\
(n=60)\end{array}$ \\
\hline L2-L4 to age & $-0.34^{*}$ & $\mathrm{NS}$ & $\mathrm{NS}$ \\
FN to age & $-0.28^{*}$ & $\mathrm{NS}$ & $\mathrm{NS}$ \\
L2-L4 to AAM & $\mathrm{NS}$ & $\mathrm{NS}$ & $\mathrm{NS}$ \\
FN to AAM & $0.24^{*}$ & $\mathrm{NS}$ & $\mathrm{NS}$ \\
L2-L4 to YSM & $\mathrm{NS}$ & $\mathrm{NS}$ & $-0.27^{*}$ \\
FN to YSM & $-0.28^{*}$ & $\mathrm{NS}$ & $-0.33^{\star *}$ \\
L2-L4 to BMI $\left(\mathrm{kg} / \mathrm{m}^{2}\right)$ & $\mathrm{NS}$ & $0.41^{*}$ & $0.48^{\star \star *}$ \\
FN to BMI $\left(\mathrm{kg} / \mathrm{m}^{2}\right)$ & $\mathrm{NS}$ & $\mathrm{NS}$ & $0.39^{\star *}$ \\
L2-L4 to duration & $\mathrm{NS}$ & $\mathrm{NS}$ & $\mathrm{NS}$ \\
FN to duration & $\mathrm{NS}$ & $\mathrm{NS}$ & $\mathrm{NS}$ \\
L2-L4 to HbA $\mathrm{C}$ & $\mathrm{NS}$ & $0.36^{*}$ & $\mathrm{NS}$ \\
FN to HbA ${ }_{1} \mathrm{C}$ & $\mathrm{NS}$ & $\mathrm{NS}$ & $\mathrm{NS}$ \\
\hline
\end{tabular}

AAM, age at menopause; YSM, years elapsed since menopause; NS, not significant.

${ }^{\star} P<0.05,{ }^{\star \star} P<0.01,{ }^{\star \star \star} P<0.001$.

alone appeared to explain 53\% of FN BMD variance. In D-NMP women, BMI and chronological age in common explained $66 \%$ of vertebral BMD variance, whereas BMI together with the age at menopause explained $64 \%$ of the FN BMD variance. The duration of diabetes was not shown as a participating factor in any of the aforementioned multiple regression models (Table 5).

\section{Discussion}

Premature menopause constitutes a risk factor for osteoporosis, whereas the influence of type 2 diabetes on bone mass is described by non-uniform findings $(2-4)$. In several studies, women after spontaneous premature menopause exhibited decreased BMD values of trabecular bone compared with women after normal menopause and of similar age (13-14). In an experimental model, additive adverse effects of oestrogen deprivation after oophorectomy combined with a diabetic state have been described (23). In this context, it was of particular interest to investigate the effect of the co-existence of these two independent conditions on the skeletal metabolism.

In the present study, vertebral and FN BMD values of diabetic women with premature menopause were compared with the respective values of either non-diabetic premature postmenopausal women or diabetic normal postmenopausal women who served as control groups. Based on the aforementioned studies regarding healthy subjects, the rationale of performing both comparisons was to reliably clarify the effect of diabetes on bone mass. We did not include in the study a third control group of postmenopausal non-diabetic women (NMP) since, in contrast to all other groups, NMP women would differ from the studied D-EMP ones in two parameters (diabetes existence and type of menopause), so that the extracted results could not be attributed to 
Table 5 Stepwise multiple regression analysis presenting the degree to which each of the anthropometric or diabetes parameters explains significantly the BMD variation. Diabetes duration absent in any model.

\begin{tabular}{|c|c|c|c|c|c|c|c|c|}
\hline Group & Site & Age & AAM & YSM & BMI & $\mathrm{HbA}_{1} \mathrm{c}$ & $\mathbf{R}^{2}(\operatorname{adj})$ & D-W \\
\hline \multirow[t]{2}{*}{ D-EMP } & L2-L4 & $\begin{array}{l}0.008^{\star \star *} \\
(3.55)\end{array}$ & & & & $\begin{array}{l}0.065^{\star \star} \\
(3.12)\end{array}$ & 0.68 & 1.77 \\
\hline & $\mathrm{FN}$ & $\begin{array}{l}0.007^{\star *} \\
(2.8)\end{array}$ & & & & $\begin{array}{l}0.047^{*} \\
(2.1)\end{array}$ & 0.67 & 2.17 \\
\hline \multirow[t]{2}{*}{ EMP } & L2-L4 & $\begin{array}{l}0.02^{\star \star \star} \\
(9.2)\end{array}$ & & $\begin{array}{l}-0.018^{\star \star} \\
(-2.6)\end{array}$ & & & 0.6 & 1.84 \\
\hline & $\mathrm{FN}$ & & $\begin{array}{l}0.017^{\star \star \star} \\
(58.2)\end{array}$ & & & & 0.53 & 2.05 \\
\hline \multirow[t]{2}{*}{ D-NMP } & L2-L4 & $\begin{array}{l}0.007^{\star \star \star} \\
(4.67)\end{array}$ & & & $\begin{array}{l}0.016^{\star \star \star} \\
(4.88)\end{array}$ & & 0.66 & 2.01 \\
\hline & $\mathrm{FN}$ & & $\begin{array}{l}0.011^{\star \star \star} \\
(5.9)\end{array}$ & & $\begin{array}{l}0.009^{\star *} \\
(2.77)\end{array}$ & & 0.64 & 2.02 \\
\hline
\end{tabular}

AAM, age at menopause; YSM, years since menopause, $R^{2}$ (adj), $R^{2}$ adjusted value; D-W, Durbin-Watson statistic

${ }^{\star} P<0.05,{ }^{\star \star} P<0.01,{ }^{\star \star \star} P<0.001$ (two tailed test); $t$-statistic in parentheses.

diabetes alone. Such an approach might complicate the issue and lead to an irrelevant comparison between D-NMP and NMP. All women were selected with a postmenopausal interval greater than 10 years. Therefore, we were able to observe the effect of diabetes long after the state of high bone turnover that is inherent in the early postmenopausal period.

Vertebral BMD values of diabetic women with premature menopause were significantly greater than those of premature menopausal women but without diabetes. This transpired despite the slightly older mean age of the D-EMP group, suggesting a protective effect of the diabetic state on bone mass status. This is further corroborated by the lack of any significant difference of vertebral BMD between the premature menopausal and the normal menopausal diabetic groups, in contrast to that observed in healthy subjects $(13,24)$. Trabecular bone, which is metabolically more active than mixed bone, probably profits to a greater extent from the diabetic state in the premature menopausal woman. In this study, the positive effect of diabetes observed on vertebral bone mass cannot be attributed to obesity (which is known to favour bone mass), since the BMI values between the diabetic and the non-diabetic premature menopausal women did not differ significantly. Cases of diabetic women with diffuse inter-vertebral skeletal hyperostosis were excluded, as this lesion can falsely increase the vertebral BMD measured by DXA.

With regards to the mixed cortical-trabecular bone as found in the femoral neck, no difference in absolute BMD values was observed between the two diabetic groups. This implies that despite the younger age at menopause and the greater interval of time that had elapsed since menopause in the D-EMP women, they did not suffer the expected increased cumulative bone loss. The ageadjusted femoral neck BMD values (Z scores) of D-EMP women were significantly higher than those of EMP women. The slight difference in mean age (almost 1 year) could account for this significant difference in $\mathrm{Z}$ score but not in the absolute BMD values of femoral neck between the same groups.

The length of time elapsed since menopause does not appear to negatively affect the bone mass of either type of bone in the women with diabetes and premature menopause, in contrast to the diabetic women with normal menopause and the prematurely menopausal healthy women. In addition, age only seemed to negatively affect the BMD of both anatomic sites in the nondiabetic women. This might imply that in diabetic women with premature menopause, the disturbed carbohydrate homeostasis may compensate for the negative effect of advancing age or postmenopausal period on bone mass. A further interesting finding was the lack of a positive influence of BMI expected on the bone mass of the non-diabetic women with premature menopause, as observed in both diabetic groups. It could therefore be assumed that another agent inherent in diabetic women might be responsible for the positive influence of obesity on bone mass (insulin resistance and/or hyperinsulinaemia). $\mathrm{HbA}_{1 \mathrm{c}}$ by itself, or in combination with chronological age, appears to be positively correlated with BMD of either type of bone only in the D-EMP group, despite the fact that $\mathrm{HbA}_{1 \mathrm{c}}$ levels were not remarkably abnormal. It appears that there is a positive relation between glucose levels and the protective effect of diabetes on bone mass. This is in accordance with previous investigators, who implicate insulin levels in bone mass preservation $(16,25)$. The levels of insulin growth factors (IGFs) have also been implicated in these mechanisms (17). Unfortunately, immunoreactive insulin or IGF levels were not available, so that the favourable effects of hyperglycaemia on bone mass cannot be attributed with certainty to possible concomitant hyperinsulinaemia. It should, of course, be taken into consideration that $\mathrm{HbA}_{1 \mathrm{c}}$ levels reflect glucose control of the previous 2 months only and in all probability do not accurately represent the level of glucose control over several years of active bone metabolism. 
A further interesting finding was the lack of any relation of bone mass to disease duration in both diabetic groups. In all likelihood this implies that diabetes has a protective rather than an anabolic effect on bone tissue. Diabetic women were included in the study, provided that the reported disease duration exceeded $3 / 4$ of the postmenopausal period. Most of these women were not diabetic at the time of the menopause - however, impaired glucose intolerance cannot be ruled out. This subgroup of diabetic women lacked the presumed protective effect of the disease during the early postmenopausal period and the inclusion of such women in both diabetic groups probably leads to an underestimation of this favourable effect of diabetes on bone mass when compared with non-diabetic women. Unfortunately, the small number of D-EMP women who were diagnosed before their menopausal age did not allow a further investigation of this particular issue. However, despite the protective effect of diabetes on measured bone mass as proposed by this study, a similar effect on fracture risk cannot be positively suggested since fracture incidence was not monitored.

In summary, the present study indicates that diabetic women after premature menopause exhibit significantly higher BMD values compared with prematurely menopausal women without diabetes in the metabolically more active trabecular bone. The mixed bone BMD values do not differ significantly between the same groups. No significant differences were observed between the BMD values of diabetic women, either after premature or normal menopause. The diabetic state, rather than the disease duration, appears to account for the favourable influence observed on bone mass.

\section{Acknowledgements}

This study was feasible due to the support of The Alexander von Humboldt Foundation, Bonn, Germany and the Research Grant Authority of Athens University.

\section{References}

1 Isaia G, Bodrato L, Carlevatto V, Mussetta M, Salamano G \& Molinatti G. Osteoporosis in type 2 diabetes. Acta Diabetologica Latina $1987 \mathbf{2 4} 305-310$.

2 Tuominen JT, Impivaara O, Puukka $\mathrm{P} \&$ Ronnemaa T. Bone mineral density in patients with type 1 and type 2 diabetes. Diabetes Care 199922 1196-2000.

3 Weinstock RS, Goland RS, Shane E, Clemens T, Lindsay R \& Bilezekian JP. Bone mineral density in women with type 2 diabetes mellitus. Journal of Bone and Mineral Research 1989 4 97-102.

4 van Daele P, Stolk R, Burger H, Algra D, Grobbee D, Hofman A, Hofman A, Birkenhäger JC \& Pols H. Bone density in non-insulin-dependent diabetes mellitus. The Rotterdam study. Annals of Internal Medicine $1995 \mathbf{1 2 2}$ 409-414.

5 Nicodemus KK \& Folsom AR. Iowa Women's Health Study. Type 1 and type 2 diabetes and incident hip fractures in postmenopausal women. Diabetes Care 200124 1192-1197.
6 Schwartz AV, Sellmeyer DE, Ensrud KE, Cauley JA, Tabor HK, Schreiner PJ, Jamal SA, Black DM \& Cummings SR. Older women with diabetes have an increased risk of fracture: a prospective study. Journal of Clinical Endocrinology and Metabolism $20018632-38$.

7 Heath H III, Melton LJ III \& Chu CCP. Diabetes mellitus and risk of skeletal fracture. New England Journal of Medicine $1980 \mathbf{3 0 3}$ 567-570.

8 Matkovic V. Calcium metabolism and calcium requirements during skeletal modelling and consolidation. American Journal of Clinical Nutrition $1991 \mathbf{5 4} 245-260$.

9 Frost HM. The role of changes in mechanical usage set point in the pathogenesis of osteoporosis. Journal of Bone and Mineral Research 19927 253-261.

10 Riggs BL \& Melton LJ III. Involutional osteoporosis. New England Journal of Medicine $1986 \mathbf{3 1 4} 1676-1686$.

11 Nordin BEC, Need AG, Chatterton BE, Horowitz M \& Morris HA. The relative contributions of age and years since menopause to postmenopausal bone loss. Journal of Clinical Endocrinology and Metabolism $1990 \mathbf{7 0} 83-88$.

12 Bjarnason K, Hassager C, Ravn P \& Christiansen C. Early postmenopausal diminution of forearm and spinal bone mineral density: a cross-sectional study. Osteoporosis International 19955 35-38.

13 Hadjidakis D, Kokkinakis E, Sfakianakis M \& Raptis SA. The type and time of menopause as decisive factors for bone mass changes. European Journal of Clinical Investigation 199929 877-885.

14 Hadjidakis D, Kokkinakis E, Sfakianakis M \& Raptis SA. Bone density patterns after normal and premature menopause. Maturitas $2003 \mathbf{4 4 2 7 9 - 2 8 6 .}$

15 Vega EM, Egea MA \& Mautalen CA. Influence of the menopausal age on the severity of osteoporosis in women with vertebral fractures. Maturitas $199419117-124$.

16 Barrett-Connor E \& Kritz-Silverstein D. Does hyperinsulinemia preserve bone? Diabetes Care $1996191388-1392$.

17 Jehle PM, Jehle DR, Mohan S \& Bohm BO. Serum levels of insulinlike growth factor system components and relationship to bone metabolism in type 1 and type 2 diabetes mellitus patients. Journal of Endocrinology 1998159 297-306.

18 Piepcorn B, Kann P, Forst T, Andreas J, Pfutzner A \& Beyer J. Bone mineral density and bone metabolism in diabetes mellitus. Hormone and Metabolic Research 199729 584-591.

19 Dorman JC, Steenkiste AR, Foley TP, Strotmeyer ES, Burke JP, Kuller LH \& Kwoh CK. Menopause in type 1 diabetic women: is it premature? Diabetes 200150 1857-1862.

20 Lopez-Lopez R, Huerta R \& Malacara JM. Age at menopause in women with type 2 diabetes mellitus. Menopause 19996 $174-178$.

21 Hadjidakis D, Kokkinakis E, Giannopoulos G, Merakos G \& Raptis SA. Bone mineral density of vertebrae, proximal femur and os calcis in normal Greek subjects as assessed by dual-energy X-ray absorptiometry: comparison with other populations. European Journal of Clinical Investigation 199727 219-227.

22 World Health Organization. Assessment of fracture risk and its application to screening for postmenopausal osteoporosis, WHO Technical Report Series 843. Geneva: WHO, 1994.

23 Fukuharu M, Sato J, Ohsawa I, Oshida Y, Nagasaki M, Nakai N, Shimomura Y, Hattori M, Tokudome S \& Sato Y. Additive effects of oestrogen deficiency and diabetes on bone mineral density in rats. Diabetes Research and Clinical Practice $2000481-8$.

24 Pouilles JM, Tremollieres F, Bonneu M \& Ribot C. Influence of early age at menopause on vertebral bone mass. Journal of Bone and Mineral Research 199423 311-315.

25 Barrett-Connor E \& Holbrook TL. Sex differences in osteoporosis in older adults with non-insulin-dependent diabetes mellitus. Journal of the American Medical Association $1992 \mathbf{2 6 8}$ $3333-3337$.

Received 26 July 2004

Accepted 16 November 2004 